\title{
11. Analyse du discours économique dans le cadre d'une linguistique distributionnelle et transformationnelle
}

Isabelle Foltête

\section{CpenEdition}

\section{Journals}

Édition électronique

URL : https://journals.openedition.org/ml/1417

DOI : $10.4000 / \mathrm{ml} .1417$

ISSN : 2274-0511

Éditeur

Association Modèles linguistiques

\section{Édition imprimée}

Date de publication : 1 juillet 1999

Pagination : 119-134

\section{Référence électronique}

Isabelle Foltête, «11. Analyse du discours économique dans le cadre d'une linguistique

distributionnelle et transformationnelle », Modèles linguistiques [En ligne], 40 | 1999, mis en ligne le 01

mai 2017, consulté le 01 juillet 2021. URL : http://journals.openedition.org/ml/1417 ; DOI : https:// doi.org/10.4000/ml.1417

Ce document a été généré automatiquement le 1 juillet 2021.

(C) Modèles Linguistiques 


\title{
11. Analyse du discours économique dans le cadre d'une linguistique distributionnelle et transformationnelle
}

\author{
Isabelle Foltête
}

\section{Introduction}

1 La presse économique est l'une des presses spécialisées les plus importantes actuellement et elle fait l'objet de nombreuses investigations, particulièrement de la part des spécialistes de l'information et de la communication. Le discours économique étant un de nos domaines de recherche, nous avons choisi pour l'analyser de nous situer dans le cadre théorique de la grammaire harrissienne développée en France par l'équipe de linguistes du L.A.D.L ${ }^{1}$. Cet article est donc une illustration de ce que l'approche distributionnelle et transformationnelle peut apporter de précision et de rigueur à la description de ce discours.

2 Après avoir défini quelques données, dont le cadre théorique, nous montrerons comment des prédicats très récurrents et spécifiques du domaine de l'économie déterminent la dynamique de ce type de discours et font apparaître les réseaux de relations qui rendent compte des places et des rôles des acteurs et des composantes de ce domaine. Puis, nous observerons quels sont les changements qui surviennent, lorsque nous opérons certaines manipulations sur les prédicats. 


\section{Données préalables}

\subsection{Le corpus}

3 Notre corpus est composé d'articles de journaux, quotidiens et hebdomadaires (dont Le Figaro-économie, Le Monde de l'économie, Les Echos, La Tribune, L'Expansion, Capital...) qui sont lus par les dirigeants et cadres d'entreprises, les économistes, les étudiants qui se spécialisent dans ce domaine et tous les gens qui s'intéressent à l'économie.

\subsection{Le discours de l'économie}

Si le terme économie signifie l'ensemble des faits relatifs à la production, à la distribution et à la consommation des richesses dans une collectivité humaine (Petit Robert), cela implique des échanges de biens, de services et de monnaie entre les acteurs du circuit économique entreprises, banques et consommateurs - l'objectif prioritaire étant la croissance. Nous pouvons alors nous attendre à ce que, dans la presse, l'accent soit mis sur les quantités, les valeurs, la croissance et les moyens d'y parvenir.

5 Le discours de l'économie qui, comme tout discours spécialisé, fait appel à un vocabulaire spécifique et présente des fréquences élevées de mots "techniques", s'adresse à une communauté homogène de lecteurs. La communauté de la presse économique en France est composée des économistes, des cadres d'entreprises et des journalistes qui sortent des mêmes écoles et des mêmes universités, fréquentées par des étudiants qui prendront le relais des premiers. Cette presse est plus qu'une source d'informations pour les spécialistes, elle constitue un véritable outil de travail.

\subsection{Cadre théorique}

6 Même si nous ne reproduisons pas telle quelle l'analyse harrissienne de Discourse Analysis (1959), les principes de la théorie distributionnelle et transformationnelle de Z.S. Harris restent le fondement de notre recherche. Pour affiner notre analyse, nous avons utilisé les outils élaborés au L.A.D.L. et au L.L.I. ${ }^{2}$. Résumons-les.

7 Z.S. Harris a décrit la phrase élémentaire en termes d'opérateurs (ou prédicats) et d'arguments, le sens de l'opérateur étant déterminé par la sélection de ses arguments, et donné les règles de fonctionnement de la langue ; son objectif était d'analyser non seulement les structures formelles, mais "aussi les séquences de mots réelles qui constituent les phrases réelles de la langue » (1976). C'est dans cette optique que le L.A.D.L. a construit, pour l'étude systématique du lexique français, un lexiquegrammaire qui a pour ambition de rendre compte de l'emploi des mots dans le cadre des structures de phrases et qui donne les propriétés syntaxiques des différentes catégories lexicales, sur la base des emplois de prédicats. Dans un premier temps, les arguments des verbes ont été classés en fonction des critères distributionnels suivants : humain, non humain, concret, non restreint. Puis, considérés comme insuffisants, ils ont été complétés par des propriétés telles que pluriel pour les constructions exigeant un sujet ou un complément au pluriel, $N p c$ (substantif "partie du corps») et éventuellement " partie de l'objet » (Leclère, 1990).

G. Gross, qui travaille dans le même cadre théorique d'un lexique-grammaire, constatant lui aussi que "les opérateurs sont très largement polysémiques ", ce qui 
pose des problèmes pour la description des classes, a alors entrepris de rechercher des informations supplémentaires concernant les arguments et il a sous-catégorisé les traits en sous-classes sémantiques, les «classes d'objets " qui se sont révélées assez précises pour permettre de discriminer le sens des opérateurs. Les classes d'objets sont élaborées à partir des distributions et chaque classe est définie par un ensemble typique d'environnement. Prenons comme exemple le verbe passer à sujet humain. Il ne suffit pas de dire que les objets peuvent être humain, abstrait, locatif ou concret, pour décrire les emplois. Le trait humain étant trop riche, il faudra diviser les humains en sous-classes: <grade> pour passer capitaine (domaine militaire), <professeur> pour passer professeur (domaine universitaire), <fonction> pour passer directeur. Lorsque l'objet est un abstrait, nous aurons recours à la classe des événements <examen, concours> pour passer le bac, passer le concours des Mines... <fêtes> pour passer Noël (à la campagne). Les sous-ensembles définis ci-dessus (grade, professeur, fonction, examen, concours, fêtes) constituent des classes d'objets. Si l'on veut obtenir l'ensemble des emplois de passer, on devra recenser les classes d'objets qui caractérisent l'objet temps (passer les vacances, la soirée), l'objet locatif (passer le seuil de la porte, la rivière)..., puis les différentes classes de sujets (cette couleur passe, le temps passe vite...). Quant aux propriétés transformationnelles, elles vont dépendre des différents emplois de l'opérateur. Les phrases dérivées, construites autour de tel emploi spécifique du verbe, seront étudiées à partir de la structure de base. Pour la description des substantifs prédicatifs - dont les déverbaux - des adjectifs..., le L.A.D.L. procède de la même façon que pour les verbes; il les analyse dans le cadre des structures de phrases. Ces prédicats sont définis par leur domaine d'arguments et par leur actualisation. Parmi les actualisateurs, nous ne retiendrons ici que les verbes supports dont nous rappelons la définition :

Les verbes supports sont des verbes prédicativement vides qui servent à actualiser les prédicats nominaux, ils prennent donc en charge les informations de personne, de temps et de nombre attribuées à l'élément prédicatif.

Pour l'étude des groupes nominaux prédicatifs, nous partons donc des phrases à verbe support. A partir de la phrase (1), nous allons suivre les étapes de la nominalisation :

(1) Les ventes de skis Salomon ont fortement chuté au Japon

[Nominalisation] = Les ventes de skis Salomon ont enregistré une forte chute au Japon

[Relativation] = La forte chute que les ventes de skis Salomon ont enregistrée au Japon

[Réduction] = La forte chute des ventes de skis Salomon au Japon

Dans notre cadre, les nominalisations ne sont pas considérées comme des transformations de phrases (verbales) en syntagmes nominaux $(\mathrm{P} \rightarrow \mathrm{SN})$ mais comme des relations transformationnelles non orientées entre deux phrases (Harris 1976).

\subsection{La démarche d'analyse}

11 Dans la presse économique, nous pouvons facilement repérer, à la première lecture, des structures très récurrentes qui présentent des données quantifiées et dont les arguments appartiennent à des classes spécifiques et homogènes du domaine de l'économie. En nous inspirant du cadre théorique exposé ci-dessus, nous avons répertorié dans notre corpus les prédicats, exprimant la mesure, dont les arguments pouvaient être regroupés dans des « classes d'objets ». 

présenter sous deux formes :

(3a) Les exportations japonaises vers les Etats-Unis ont augmenté de 3 milliards de US\$

(3b) Le Japon a augmenté ses exportations vers les Etats-Unis de 3 milliards de US\$

Nous en présentons ici deux types, le premier donne la mesure des marchés, des échanges, des données relatives aux entreprises et des indicateurs de l'économie :

(2) (La reprise est bien amorcée...) La croissance pourrait être de 2,5\% cette année

Le deuxième, qui indique les variations de toutes les données citées ci-dessus, peut se Nous reviendrons plus en détail sur leur constitution en 2.2.

Ces prédicats (2) et (3), particulièrement le premier, sont généralement précédés d'une fonction du sens du substantif prédicatif dérivé et de son sujet.

\section{Analyse proprement dite}

\section{1. Prédicats de mesure et appréciation} phrase appréciative dont le but est d'évaluer la donnée chiffrée, de façon plus ou moins explicite, sur une échelle qui va de "très bon " à "très mauvais ". La reprise est bien amorcée, qui précède le prédicat de mesure (2), entretient une relation sémantique avec celui-ci dans le sens où la croissance est un indicateur de la reprise économique. L'appréciation positive (reprise bien amorcée) se voit confirmée par le «bon » chiffre $(2,5 \%)$ de la croissance.

Nous examinerons en 2.1. les relations qui existent entre l'appréciation et la mesure. des arguments et les déplacements qu'ils subissent, de rendre compte des places qui sont données aux différents actants dans le procès, et de la façon dont ils interviennent sur celui-ci. Dans la phrase à verbe intransitif :

(4) Les ventes de Peugeot ont fortement baissé

le sujet non humain est « intérieur » au procès et dans son rôle de " patient », il subit le procès, ce qui signifie que la société Peugeot n'a pas d'emprise sur l'état de ses ventes, qui sont soumises aux lois du marché et à la conjoncture, alors que dans la phrase :

(5) France Télécom va encore baisser ses tarifs sur le procès est maximum et que l'« activité indépendante » de l'objet est minimum, autrement dit, que c'est la société qui a pris la décision de baisser ses tarifs, et que l'on a affaire ici à un acte " volontaire ».

Nous allons d'abord analyser les phrases verbales de mesure et de variation, puis les nominalisations par verbes supports, afin de voir comment ils sont sélectionnés en Reprenons la phrase (2) :

(2) La croissance pourrait être de 2,5\% cette année

Elle est composée du verbe support (être), de la préposition (de) et du complément de mesure $(2,5 \%)$ dont l'unité de mesure est contrainte par le groupe nominal sujet. Les substantifs en position sujet appartiennent à des classes d'objets qui rassemblent les acteurs du circuit économique, les types de marchés et d'échanges, les données relatives aux entreprises, les produits, les services et les monnaies, les indicateurs de 
conjoncture..., que l'on peut coder en utilisant un certain nombre d'informations. Comme tous les substantifs ne sont pas identiques du point de vue syntaxique, nous devons séparer les substantifs prédicatifs (qui ont la même extension que les verbes qui leur sont associés) et les arguments élémentaires (substantifs qui n'ont pas eux-mêmes d'arguments). Pour la description des substantifs, nous prendrons en compte les informations suivantes:

- $\mathrm{T}$ désigne les traits de nature syntactico-sémantiques comme: humains (hum), inanimé concret (inc), inanimé abstrait (ina), locatif (loc)...,

$24-\mathrm{C}$ correspond aux classes d'objets désignant des ensembles sémantiques homogènes (unités de mesure, moyens de transport, véhicules, vêtements...),

25 - D indique le domaine dans lequel le substantif en question prend sa signification (économie, (secteur) automobile, commerce...).

26 Pour les substantifs prédicatifs, nous préciserons l'opérateur associé $(\mathrm{Op})$ : exportation est associé à exporter, ainsi que les arguments.

Donnons quelques exemples de codage de substantifs prédicatifs suivis des emplois correspondants :

Immatriculation/T :ina/C:unité de mesure/Op:immatriculer/No:hum/

N1:<véhicule>/D:automobile/

Le nombre des immatriculations de voitures n'a pas dépassé 100000 véhicules en

juin

Croissance/T:ina/C:changement d'état/Op:croître/N0:loc<pays,marché>/

N1:de<\%>/D :économie/

Le taux de croissance du Royaume-Uni sera d'au moins 3 \% en 1998

Vente1/T:ina/C:unitédemesure/Op:vendre/N0:hum/N1:<produits>/D:commerce/

Les ventes de Beaujolais nouveau ont atteint 6 millions de bouteilles cette année

Nous noterons la contrainte qui existe entre la nature du produit (vin de Beaujolais) et l'unité de mesure (bouteille). Vente1 correspond au substantif qui désigne le volume des ventes de produits, évaluable dans toutes les unités de mesure (ventes de pétrole: barils; ventes de blé : quintaux, tonnes; ventes de jeans: unités, pièces...). Si nous observons l'emploi suivant du substantif vente:

Les ventes de produits de luxe ont atteint 100 milliards de francs en 1997

nous avons affaire à un deuxième substantif vente qui identifie le produit des ventes, évaluable uniquement en unités monétaires, et dont l'opérateur associé est vendre pour. La phrase à opérateur :

La France a vendu pour 100 milliards de francs de produits de luxe en 1997

est le résultat de la restructuration de :

La France a vendu des produits de luxe pour 100 milliards de francs en 1997

31 Nous aurons pour ce deuxième substantif la présentation suivante :

Vente2/T:ina/C:unité monétaire/Op:vendre pour/N0:hum/N1:<produits>/

N2:pour<unité monétaire>/D:commerce/

dans laquelle il faudra indiquer la contrainte sur le déterminant indéfini des (produits de luxe) ou partitif $d u$ (pétrole).

Présentons maintenant quelques arguments élémentaires appartenant au domaine de l'automobile (ici secteur d'activité) :

Petit utilitaire/T:inc/C:véhicule/D:automobile/

Camion/T:inc/C:véhicule/D:automobile/ 
Véhicule léger/T:inc/C:véhicule/D:automobile/ supports dont le choix dépend de la nature du substantif en position sujet. Ils peuvent
être présentés sous forme de fichier. Les verbes qui suivent, sont «appropriés » à la
classe des substantifs chiffrables en unités de mesure:

Nous pourrions ajouter à cette liste : voiture de tourisme, monospace, poids lourd... qui peuvent entrer dans la phrase classificatoire : Un petit utilitaire est un véhicule, et dont l'opérateur approprié est immatriculer : 100000 monospaces ont été immatriculés en 1997.

Véhicule peut aussi figurer dans la classe des unités de mesure :

Véhicule/T:inc/C:unité de mesure/D:commerce/

Les verbes qui peuvent introduire une mesure sont d
supports dont le choix dépend de la nature du subst
être présentés sous forme de fichier. Les verbes qui
classe des substantifs chiffrables en unités de mesure
atteindre n unité de mesure/No:ina<unité de mesures

Les immatriculations de voitures neuves n'ont pas atteint 95000 véhicules en juin

s'élever à $\mathrm{n}$ unité de mesure/No:ina<unité de mesure>

Les ventes d'ordinateurs domestiques se sont élevées à (2 millions d'unités

+20000 milliards de francs)

coter $\mathrm{n}$ unité monétaire/N0:ina<actions-titres-matières premières-monnaies>

Le baril de pétrole cotait 19, 50 dollars hier à Londres

En mettant en relation les fichiers des classes d'objets en position sujet avec celui des unités de mesure et le fichier des verbes, nous obtenons la sélection des prédicats de mesure.

Dans la presse économique, ces prédicats de mesure sont juxtaposés à des phrases appréciatives à adjectif et substantif prédicatifs ou à expression figée, comme nous l'avons vu ci-dessus pour (2), qui expriment le jugement du journaliste sur la donnée chiffrée ou qui indiquent au lecteur comment il faut l'interpréter. En effet, un chiffre n'a de sens que par rapport à d'autres chiffres, et seuls les journalistes et les économistes, en tant que spécialistes, sont en mesure d'apprécier et de resituer dans son contexte toute donnée chiffrée, parce qu'ils sont en possession de tous les chiffres nécessaires au traitement de l'information économique.

Prenons l'exemple de La Tribune du 10 septembre 1997 :

ALLEMAGNE. LE CHÔMAGE CONTINUE SON INEXORABLE PROGRESSION

(6) ... Pour le président de l'office du travail, « le creux de la vague devrait avoir été atteint à l'Ouest », compte tenu de la vigueur des exportations. Les perspectives dans les nouveaux Länder, en revanche, restent sombres. Le taux de chômage en données brutes est de $18,3 \%$.

Le prédicat de mesure, l'adjectif prédicatif sombre exprime une appréciation négative. Le taux de chômage étant un des éléments constitutifs de l'évaluation des perspectives économiques d'un pays, on peut en déduire que sombre qualifie aussi la donnée chiffrée qui doit être considérée comme négative.

41 L'article de La Tribune est construit sur l'opposition (mise en relief dans la deuxième phrase par en revanche) entre : l'économie allemande est sur la bonne voie dans la première partie, avec de bons chiffres pour le PIB et la croissance (entre 2,5 et $3 \%$ ) et la progression inexorable du chômage, particulièrement à l'Est, dans la deuxième partie.

L'article du Monde (8 juillet 1997) :

LA FRANCE, UN GÉANT PARESSEUX

est construit sur une opposition un peu moins tranchée : 
(7) La France n'en est pas à un paradoxe près. D'un côté, son tourisme se porte bien. Selon les dernières statistiques... 68,4 \% de Français ont pris des vacances en 1995 et 61,5 millions d'étrangers sont venus en France en 1996...

L'autre visage est moins souriant... le nombre des vacanciers français a tendance à stagner, la consommation touristique des Français en France progresse faiblement, la croissance du tourisme international en France est deux fois moins importante que celle du tourisme international dans le monde. prédicatifs qui donnent une appréciation, de type quantitatif, positive ou négative. Quant aux phrases (d), (e) et (f), elles présentent des structures différentes à prédicat nominal ; s'enfoncer dans le marasme représente l'aspect intensif d'être dans le marasme, phrase à verbe support être dont le prédicat dans le marasme exprime une appréciation d'ordre quantitatif. Afficher un certain optimisme dont le verbe support afficher est une extension du support avoir est à mettre en relation avec l'expression à verbe support et adjectif prédicatif être optimiste dont le sens est positif. Le prédicat avoir le vent en poupe est une expression figée (aucun élément ne peut varier) qui donne aussi une appréciation positive. Si nous ne nous attardons que sur les deux dernières phrases (e) et (f), nous constatons que l'appréciation est formulée en fonction de facteurs extérieurs au discours (que nous ignorons), c'est-à-dire que le journaliste se réfère constamment à la réalité de la vie économique. En effet, l'optimisme qu'affiche le BTP (Bâtiment-Travaux-Publics) ne peut se comprendre que par rapport à la crise des années passées; quant à L'Oréal, numéro un mondial des cosmétiques, c'est actuellement le modèle de l'entreprise qui réussit. 


\subsection{Les prédicats de variation}

d'objets qui ont été décrites en 2.1.: les acteurs du circuit économique, les types de marchés et d'échanges, les données relatives aux entreprises, les produits, les services et les monnaies, les indicateurs de conjoncture... Ils n'exercent aucune action sur le procès, ils dépendent de la conjoncture. Considérons maintenant le couple de phrases :

(12) Le bénéfice semestriel d'Essilor (a augmenté + s'est accru) de $5 \%$ à 294 MF

(12a) Essilor a (augmenté + accru) son bénéfice semestriel de $5 \%$ à $294 \mathrm{MF}$ éclater » le groupe nominal sujet $\mathrm{Na}$ de $\mathrm{Nb}$, dans lequel $\mathrm{Nb}$ est complément de nom de $\mathrm{Na}$, en deux parties structurellement indépendantes (Guillet, Leclère 1981). Pour (12) = (12a), elle a la forme :

$57 \quad(\mathrm{Na}$ de $\mathrm{Nb}) \mathrm{V}$ W (suite de compléments) = Nb V Poss Na $\mathrm{W}$ de Essilor qui appartient à la classe des noms propres d'entreprises. Dans cette position, 
Essilor est un substantif non humain qui ne sert qu'à caractériser bénéfice. Le sujet de la phrase n'exerce aucune action sur le procès.

- dans (12a), le substantif bénéfice est objet du verbe transitif, précédé du possessif coréférent à Essilor devenu sujet du verbe. C'est, dans cette position, un substantif collectif humain qui exerce une action volontaire sur le procès (augmentation des bénéfices), comme nous l'avons vu en 1.4. pour la phrase (5), Cette action, qui peut être l'application d'une nouvelle stratégie, la sortie d'un nouveau produit... est évoquée dans le texte.

Bien que (12) et (12a) soient sémantiquement équivalentes, nous constatons qu'elles expriment le procès de façon différente, en introduisant une opposition entre les notions de « volontaire » et d' « involontaire ». Cette opposition est déterminante pour l'expression des données chiffrées et les journalistes l'utilisent fréquemment, particulièrement dans les titres des articles, car ils doivent donner une image positive, ou la moins négative possible, des entreprises qui constituent leur lectorat. Le nom de l'entreprise est en position sujet lorsque le résultat ou la stratégie sont jugés positifs :

(13) Générali a doublé ses profits l'an dernier Deutsche Telekom prévoit de doubler son résultat cette année

Volkswagen Belgique réduit le temps de travail en préservant l'emploi

Dans l'exemple suivant, en ne respectant pas cette règle, le journaliste veut mettre en avant le sens positif de la variation et l'importance du chiffre :

(14) Hausse de $60 \%$ du bénéfice semestriel de Bertrand Faure

Nous reconnaissons dans ce titre une nominalisation. Celle-ci se fait à l'aide de la relativation et de l'effacement du verbe support et elle est caractérisée par l'absence d'actualisation puisqu'il y a eu effacement du verbe support. Nous aurons pour (14) les étapes suivantes :

Le bénéfice semestriel de Bertrand Faure a enregistré une hausse de $60 \%$

= La hausse de $60 \%$ que le bénéfice semestriel de Bertrand Faure a enregistrée

= La hausse de $60 \%$ du bénéfice semestriel de Bertrand Faure

Le GN peut, sous cette forme, servir d'argument à un autre prédicat, mais lorsqu'il est utilisé comme titre, il l'est sans déterminant devant le substantif prédicatif hausse. Pour expliquer ce phénomène, il faut rappeler que le déterminant est avec le verbe support l'élément d'actualisation des prédicats nominaux et que le déterminant est dépendant $d u$ verbe support. Le fait d'effacer ce dernier affecte nécessairement la détermination, autrement dit, l'effacement du verbe support a entraîné l'effacement du déterminant devant le substantif prédicatif du GN utilisé comme phrase nominale indépendante.

Lorsque le résultat est négatif, le nom de l'entreprise apparaît en deuxième position :

(15) Le déficit de la MGM s'est encore creusé ou comme sujet, entretenant une relation non active dans une phrase nominalisée :

(16) Danone enregistre un tassement de sa marge opérationnelle

ce qui a pour effet de déresponsabiliser cette entreprise. On peut cependant remarquer que dans le corps de l'article, les journalistes prennent moins de précautions. Précisons que le verbe support enregistrer s'emploie avec la classe des substantifs prédicatifs qui indiquent un changement d'état (tassement, baisse, croissance...). 


\subsubsection{Le passif} substantifs, et d'en écarter d'autres. Dans l'exemple qui suit :

(17) LE TAUX DE LA CSG AUGMENTERA DE 3 OU 4 POINTS DÈS 1998

Revenus de l'épargne. Alain Juppé avait franchi le premier pas l'an dernier. La part salariale des cotisations d'assurance maladie avait été ramenée de 6,8 \% à 5,5\% du salaire brut. En contrepartie, la CSG élargie à la quasi-totalité des revenus de l'épargne avait été augmentée d'un point, ce dernier étant déductible du revenu imposable.

... Comme Jacques Chirac en 1995, le PS cherche à alléger les taxes pesant sur les revenus du travail, en alourdissant celles pesant sur le revenu du capital. La CSG remplit ce rôle puisqu'elle frappe les revenus de l'épargne à raison de 5 milliards par point. Au terme du processus, les prélèvements obligatoires sur les revenus de l'épargne auront été accrus de 20 milliards. Tandis que les cotisations salariales auront été allégées de 28 milliards. (La Tribune. 10 septembre 1997)

les sujets des passifs sont les substantifs chiffrables qui subissent les variations. Les agents humains des passifs (les dirigeants) responsables de ces variations sont effacés, ce qui signifie que le journaliste ne veut pas les nommer, c'est-à-dire qu'il ne veut pas leur imputer la responsabilité des mesures prises. Par contre, l'action d'alléger qui est populaire est attribuée à des sujets actifs (J. Chirac, le PS).

D'autres opérations vont de façon plus évidente changer la place et le rôle des actants : l'application des opérateurs faire et voir.

\subsubsection{L'opérateur causatif faire}

L'application du causatif faire à la phrase Les immatriculations des grosses cylindrées chutent, permet d'exprimer l'intervention indirecte d'un agent extérieur sur le procès :

(18) Une hausse du prix du gas-oil risque de faire chuter les immatriculations des grosses cylindrées

Les substantifs susceptibles d'être sujets du causatif constituent des classes d'événements et de phénomènes économiques (hausse du pétrole, baisse du dollar, inflation...), d'événements commerciaux (succès d'un produit, d'une campagne, application d'une stratégie...), politiques (la guerre du Golfe, l'embargo sur le pétrole irakien...), des classes d'humains (dirigeant, ministre...). Ces classes doivent être définies pour aider à l'interprétation de phrases causatives qui ne comportent pas faire, mais des verbes à deux emplois dont la forme transitive peut être utilisée comme causatif. Remplaçons dans (18) faire chuter par ralentir ou comprimer et comparons la phrase causative synonyme :

(18a) Une hausse du prix du gas-oil risque de (ralentir + comprimer) les immatriculations des grosses cylindrées

à la phrase active :

(19) Peugeot a décidé de (ralentir + comprimer) la production de certains modèles

nous constatons que ralentir et comprimer offrent deux interprétations différentes : dans (18a), le sujet une hausse du prix du gas-oil « extérieur au procès » agit indirectement sur celui-ci et l'objet a une "activité indépendante", alors que dans (19) le sujet humain (les dirigeants de Peugeot) agit directement sur le procès et le complément direct est sous le contrôle de ce sujet. 


\subsubsection{L'opérateur à lien voir}

Rappelons qu'un opérateur à lien «n'introduit pas de nouvel argument, car son sujet se lie à un complément de nom de la phrase » (M. Gross 1981). L'opération a la forme :

$\mathrm{Na}$ de $\mathrm{Nb} \mathrm{V}=\mathrm{Nb}$ voir Poss $\mathrm{Na} \mathrm{V}$ infinitif

L'application de cet opérateur à des phrases intransitives (Les rémunérations des salariés augmentent...; les ventes de café d'Indonésie reculent...) est très utilisée dans la presse économique. Dans les phrases :

(20) Les salariés verront leur rémunération progresser, mécaniquement, de 1,1 point

(21) L'Indonésie, troisième exportateur mondial de café, a vu ses ventes reculer à

671000 sacs en juillet contre 898909 sacs 12 mois avant

voir apporte une nuance intéressante dans le rapport agent / patient. Dans la phrase de base, salariés et Indonésie, compléments de noms, ne servent qu'à identifier respectivement rémunération et ventes de café du groupe sujet et n'ont aucun rôle dans le procès. L'opérateur voir permet de les réintroduire en première position, comme sujets de la phrase à opérateur, mais seulement comme "sujets-spectateurs » du procès (auxquels ils sont rattachés par le possessif) puisqu'ils gardent leur rôle non actif.

\subsection{Les nominalisations}

Les nominalisations par verbes supports sont couramment utilisées dans la presse économique à diverses fins. Nous allons voir le cas des phrases à verbe support, dérivées de phrases verbales.

Dérivation d'une phrase intransitive :

(22) Les immatriculations ont fortement chuté

= Les immatriculations (sont en + ont enregistré une) forte chute

Dérivation d'une phrase transitive :

(23) Smoby SA a accru son activité de $35 \%$ en 1996

= Smoby SA a connu un accroissement de 35 \% de son activité en 1996

81 Etre, enregistrer et connaître sont ici des verbes supports qui avec le substantif dérivé forment une phrase à prédicat nominal, équivalente à la phrase verbale. Les verbes supports sont nombreux et leur choix dépend de la nature sémantique du prédicat. Pour les verbes de variation, les supports être (en chute), afficher (une hausse), connaître (une progression), enregistrer (une chute) traduisent un état. Effectuer et procéder à sont utilisés pour des actions :

(24) La banque Bayerisch Vereinsbank va augmenter son capital de 3 milliards de marks pour financer l'achat d'actions de la Hypo Bank.

= La banque Bayerisch Vereinsbank va procéder à une augmentation de son capital

de 3 milliards de marks pour financer l'achat d'actions de la Hypo Bank.

82 Les nominalisations offrent la possibilité de créer des classes d'équivalences, pour exploiter toutes les possibilités d'expression d'un prédicat, et d'exprimer ou de renforcer certains aspects de ces prédicats. Dans la nominalisation suivante :

(25) Les ventes de skis Salomon ont (enregistré + subi) une forte chute au Japon ( $-50 \%$ en 4 ans) 
en choisissant subir, on veut insister sur l'aspect négatif de la variation par rapport à enregistrer qui est neutre. Entamer et poursuivre traduisent respectivement les aspects inchoatif et progressif du prédicat:

(26) Le BTP (entame + poursuit) sa remontée

Par ailleurs, en restructurant le GN sujet, on met en relief, en le déplaçant en position sujet, le substantif (Salomon) qui ne peut être que complément de nom dans la phrase de base (1) et dans la phrase dérivée (25). Restructuration du GN sujet de (25) :

(25a) Salomon (enregistre + subit) une forte chute de ses ventes de skis au Japon

Enfin, les nominalisations par verbe support permettent la constitution d'un groupe nominal par effacement du verbe support, ce qui est possible, puisqu'il est sémantiquement vide. Le groupe nominal garde le statut de phrase, comme nous l'avons vu en 1.3 pour l'exemple (1), mais de phrase non actualisée. Le GN obtenu peut devenir l'argument d'un autre prédicat :

(27) La forte chute des ventes de skis Salomon au japon a obligé le groupe à passer une provision de $66 \mathrm{MF}$

et multiplier ainsi la quantité d'information, ce qui correspond à l'objectif de la presse économique (mais aussi d'autres types de discours) qui est de " présenter des articles courts pour donner en un minimum de temps le maximum d'information » (Les Echos).

\section{Conclusion}

La description du discours économique que nous venons de présenter montre que le recours à la linguistique distributionnelle et transformationnelle peut très bien répondre aux objectifs rigoureux d'analyse d'un discours de spécialité. L'introduction des classes d'objets nous a permis de faire une description partielle mais très précise du lexique de l'économie et des prédicats les plus récurrents. Nous n'avons analysé ici que deux types de prédicats qui organisent en partie la structure du texte et qui font ressortir l'importance des éléments chiffrables et leur dépendance vis-à-vis des acteurs de l'économie (entreprises, dirigeants, pays) et des événements. D'autres, comme les prédicats d'échanges, parmi lesquels les constructions converses (donner / recevoir, allouer / toucher...), sont tout aussi pertinents pour montrer le fonctionnement des circuits économiques, les relations entre donneur (vendeur) / receveur (acheteur) / produits échangés et monnaie, représentant aussi des éléments essentiels du monde de l'économie.

\section{BIBLIOGRAPHIE}

Anscombre, J.-C., Ducrot, O. (1976), « L'argumentation dans la langue », Langages, n² 42, Paris, Larousse, p. 7-27.

Austin, J. (1970), Quand dire c'est faire, Paris, Le Seuil.

Modèles linguistiques, 40 | 1999 
Bat Zeev-Shyldkrot, H. (1984), « La concurrence entre la proposition conjonctive et voir + la proposition infinitive », The French Review, vol. LVIII, n² 2, p. 202-214.

Benveniste, E. (1974), « L'appareil formel de l'énonciation » dans Problèmes de linguistique générale 2, Paris, Gallimard, p. 79-88.

Boons, J.-P., Guillet, A., Leclère, C. (1976), La structure des phrases simples en français. I. Constructions intransitives, Genève, Droz.

Boutet, J. (1994), Construire le sens, Paris, Peter Lang.

Charaudeau, P. (1995), « Le dialogue dans un modèle de discours ", Cahiers de Linguistique française, $\mathrm{n}^{\circ}$ 17-2, Université de Genève, p. 141-173.

Dubois, J. (1978), « Présentation », Langages, n52, Paris, Larousse, p. 3-4.

Dubois, J. (1969), « Enoncé et énonciation », Langages, n 13, Paris, Larousse, p. 100-110.

Foltête, I. (1994), Analyse de la mesure dans la langue du marketing, Thèse de doctorat, Université Paris VII.

Foucault, M. (1969), L'archéologie du savoir, Paris, Gallimard.

Foucault, M. (1971), L'ordre du discours, Paris, Gallimard.

Gross, G., Vivès, R. (1986), « Les constructions nominales et l'élaboration d'un lexique-

grammaire ", Langue française, $\mathrm{n}^{\circ}$ 69, Paris, Larousse, p. 5-27.

Gross, G. (1994), « Classes d'objets et description des verbes », Langages, n 115, Paris, Larousse, p. 15-30.

Gross, G. (1994), « Un outil pour le FLE : les classes d'objets », Actes du colloque du FLE, Lille, Presses universitaires de Lille.

Gross, G. (1996), « Prédicats nominaux et compatibilité aspectuelle », Langages, n 121, Paris, Larousse, p. 54-72.

Gross, M. (1975), Méthodes en Syntaxe, Paris, Hermann.

Gross, M. (1981), « Les bases empiriques de la notion de prédicat sémantique », Langages, $\mathrm{n}^{\circ}$ 63, Paris, Larousse, p. 7-52.

Gross, M. (1986), Grammaire transformationnelle du français. Syntaxe du nom, Paris, Cantilène.

Guespin, L. (1976), «Introduction. Types de discours, ou fonctionnements discursifs ? », Langages, $\mathrm{n}^{\circ} 41$, Paris, Larousse, p. 3-11.

Guilhaumou, J. (1993), « A propos de l'analyse du discours : les historiens et le "tournant linguistique" ", Langage et société, $\mathrm{n}^{\circ}$ 65, septembre, p. 5-37.

Guillet, A. Leclère, C. (1981), « Restructuration du groupe nominal », Langages, n 63, Paris, Larousse, p. 99-125.

Harris, Z. (1969), « Discourse analysis », traduction française dans Langages, $n^{\circ} 13$, Paris, Larousse, p. 8-45.

Harris, Z. (1976), Notes du cours de syntaxe, Paris, Le Seuil.

Harris, Z. (1980), « La structure distributionnelle », traduction française dans Langages, $n^{\circ} 20$, Paris, Larousse, p. 14-34.

Joly, A., O’ Kelly, D. (1987), « Cohésion discursive et argumentation : Do dit “emphatique” en anglais contemporain », Modèles linguistiques IX : 1, Presses Universitaires de Lille, p. 93-111. 
Langages nº 55 (1979), Analyse de discours et linguistique générale, Paris, Larousse.

Langages $n^{\circ} 81$ (1986), Analyses de discours, nouveaux parcours, Paris, Larousse.

Langages n 99 (1990), Les grammaires de Harris et leurs questions, Paris, Larousse.

Langages $n^{\circ} 117$ (1995), Les analyses du discours en France, Paris, Larousse.

Leclère C. (1990). « Organisation du lexique-grammaire des verbes en français ». Langue française $\mathrm{n}^{\circ} 87$, Paris, Larousse, p. 112-122.

Lepesant, D. (1994), « Les compléments nominaux du verbe lire. Une illustration de la notion de “classe d'objets" ", Langages, $\mathrm{n}^{\circ}$ 115, Paris, Larousse, p. 31-46.

Maingueneau, D. (1991), Introduction aux lectures de l'archive, Paris, Hachette.

Maingueneau, D. (1992), « Le tour ethnolinguistique de l'analyse du discours », Langages, $\mathrm{n}^{\circ}$ 105, Paris, Larousse, p. 114-125.

Marandin, J.-M. (1976), « Problématique de l'analyse du discours française », Langages, $\mathrm{n}^{\circ} 41$, Paris, Larousse, p. 19-32.

Moirand, S. (1990), Une grammaire des textes et des dialogues, Paris, Hachette.

Moirand, S. (1996), Le discours : enjeux et perspectives. Le français dans le monde, recherches et applications, Paris, Hachette.

Pécheux, M., Fuchs, C. (1975), « Mises au point et perspectives à propos de l'analyse automatique du discours ", Langages, $n^{\circ}$ 37, Paris, Larousse, p. 7-80.

Peytard, J., Moirand, S. (1992), Discours et enseignement du français. Les lieux d'une rencontre, Paris, Hachette Références.

Roulet, E. (1991), « Vers une approche modulaire de l'analyse du discours », Cahiers de linguistique française, $\mathrm{n}^{\circ} 12$, Université de Genève, p. 53-81.

Sumpf, J., Dubois, J. (1969), « Problèmes de l'analyse du discours », Langages, n 13, Paris, Larousse, p. 3-7.

\section{NOTES}

1. Laboratoire d'Automatique Documentaire et Linguistique (CNRS), Université Paris 7.

2. Laboratoire de Linguistique Informatique, URA 1576, Université Paris 13, Villetaneuse

\section{AUTEUR}

\section{ISABELLE FOLTÊTE}

Paris X

L.L.I. (Laboratoire de Linguistique Informatique, URA 1576, Université Paris 13, Villetaneuse) 